\title{
Revisiting Domestication to Revitalize Crop Improvement: The Florigen Revolution
}

\author{
Soon Ju Park ${ }^{1 * \dagger}$, Young Koung Lee ${ }^{2 \dagger}$, Min Sung Kang ${ }^{1}$, Jong Hyang Bae ${ }^{3}$ \\ ${ }^{1}$ Division of Biological Sciences and Institute for Basic Science, Wonkwang University, Iksan 54538, Korea \\ ${ }^{2}$ Cold Spring Harbor Laboratory, Cold Spring Harbor, NY 11724, USA \\ ${ }^{3}$ Department of Horticulture Industry, Wonkwang University, Iksan 54538, Korea
}

\begin{abstract}
Most flowering plants have evolved to coordinate proper floral transition in shoot apical meristems at an appropriate time during development, referred to as "flowering time." In domesticated crops, shoot life time has been manipulated to enhance productivity via synchronizing flowering time to the local environment, thereby creating a balance between vegetative and reproductive plant architecture during the year. Rice, a typical single transitional crop, has acquired balance between prolonged tiller growth and a relatively short reproductive phase on individual shoot, resulting in increasing yield. Crops with sympodial growth, such as tomato, which undergo multiple floral transitions on the main shoot, have been artificially selected for the determination of shoot growth by investigating the dosage sensitivity of florigen activation controlling flowering time on individual shoots. Here, we summarize recent genetic-molecular studies on tomato, which have been subject to genetic manipulation of flowering time in an effort to optimize seed productivity. We also review advanced genetic approaches as potential new tools for the enhancement of crop yield by manipulating flowering time via the dosage effect of florigen.
\end{abstract}

Keywords Florigen, Shoot architecture, Dominant negative, Dosage effect, Overdominance, Tomato

\section{INTRODUCTION}

The life cycle of flowering plants is determined by changes in developmental states from leaf-forming vegetative to flower-forming reproductive apical meristems (AM) during the year (Park et al. 2014a). Flowering time is important for the reproductive success of plants, because optimization of seed production is achieved by creating a balance between vegetative and reproductive phases (Lifschitz and Eshed 2006; Nakamichi 2015).

Plants respond to daylength by sensing light and this regard have evolved molecular and genetic mechanisms within two distinct pathways, namely, the long-day and short-day responding light signaling pathways. In the model plant Arabidopsis thaliana, flowering is pro- moted under long-day conditions via regulation by the FLOWERING LOCUS T (FT) protein. Genome-wide studies on Arabidopsis ecotypes have discovered that they have adapted to growing in different regions by controlling floral transition via vernalization and long-day flowering pathways (Grillo et al. 2013; Song et al. 2013). Notably, Arabidopsis has evolved shoot apical meristems (SAM) to initiate and mainly elongate lateral branches after reproductive transition, and has thereby attained a relatively long period in the inflorescence phase (Fig. 1A). Rice, a typical short-day crop, has evolved two independent floral pathways: the Heading date 1 (Hdl, a homolog of $\mathrm{CO}$ ) pathway activates flowering under short-day conditions but suppresses flowering under long-day conditions, whereas the Early heading date 1

Received October 7, 2016; Revised November 10, 2016; Accepted November 10, 2016; Published November 30, 2016

*Corresponding authorS Soon Ju Park, sjpark75@wku.ac.kr, Tel: +82-63-850-6096, Fax: +82-63-857-8837

${ }^{\dagger}$ These authors contributed equally to this work as co-first author. 
(A)

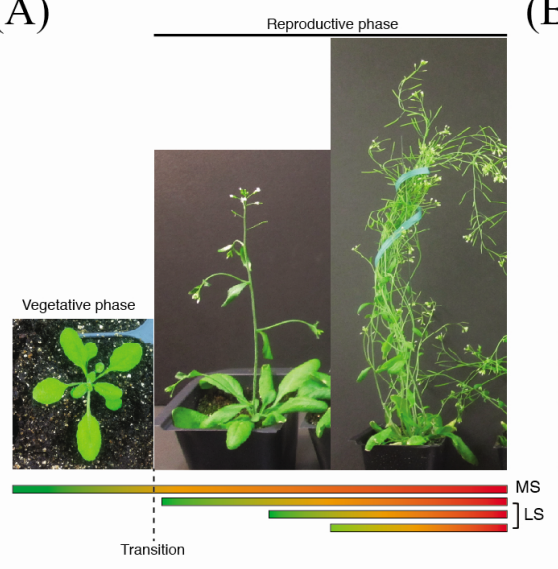

(B)

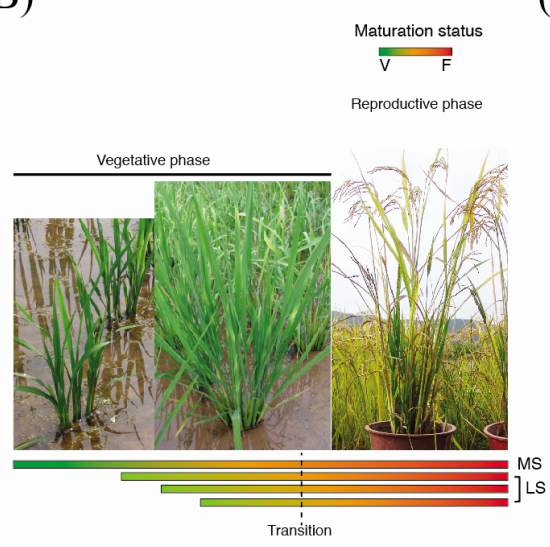

(C)

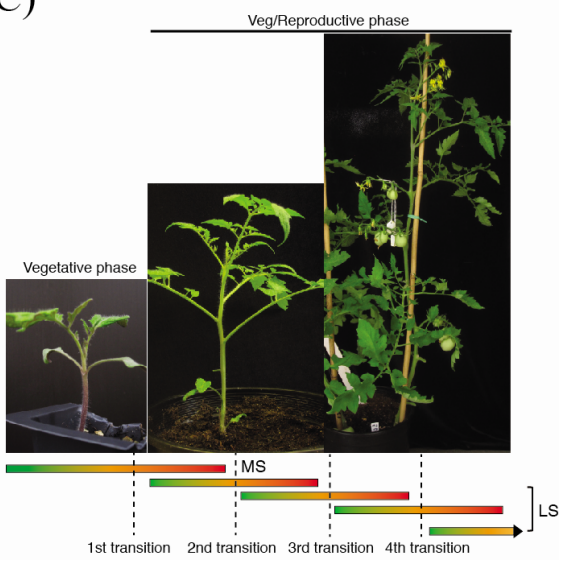

Fig. 1. Spatial and temporal development and growth of lateral shoots initiated on main shoot from vegetative to reproductive phase in Arabidopsis (A), rice (B), Tomato (C) relatively. (A) Arabidopsis, a long-day flowering plant, continuously develops and grows lateral branches on reproductive main shoots producing lateral meristems after transition. (B) Rice, a short-day flowering plant, produces lateral branches, named tillers, on main shoots before transition, since the length of vegetative phase is crucial for high yield production. (C) Primary shoot and sympodial shoots are relatively early terminated in flower. Tomato main shoot is a sum of sympodial shoots initiated on axil of last leaf on apics of main shoot, indicating indeterminate growth. Dashed lines indicate phase transition to reproductive status.

MS: main shoot, LS: lateral shoot, V: vegetative status, F: floral status.

(Ehdl) pathway accelerates flowering under both shortand long-day conditions. Integration of these pathways gives rise to the expression of $H d 3 a$ and RICE FLOWERING LOCUS T1 (RFT, rice florigens) (Izawa 2007). Interestingly, the studies on rice domestication have isolated a few key genes involved in floral transition in different regions, and have reported that different rice ecotypes prolong the period of vegetative organ development and produce many lateral tillers before floral transition (Fig. 1B) (Nakamichi 2015). Tomato, which is known as a day-neutral plant, produces flowers from AM in the primary shoot regardless of day length, and sympodial shoots are initiated in the final leaf axil of previous shoots. These shoot give rise to flowers following the production of three leaves, with reiteration of regular vegetative/reproductive transitions (Fig. 1C) (Park et al. 2012). In the tomato antiflorigen mutant "self pruning", the perennial sympodial growth has been altered to give an annual determinate sympodial growth habit, and recently, tomato florigen studies reporting $s f t /+$ heterosis and a florigen activation dosage model have suggested new techniques for post-domestication of tomato in both the field and greenhouse (Pnueli et al. 1998; Krieger et al. 2010; Jiang et al. 2013; Park et al. 2014b). Moreover, CRISPR/Cas9 genome editing technology provides breeders with an innovative technique for crop improvement, including that of edible wild species (Khatodia et al. 2016). In this review, we summarize how new discoveries on florigen pathways integrating flowering time genes have been utilized for the most recent domestications in order to improve crop yields. We also discuss how we can apply new genome editing tools to enhance the reproductive fitness of domesticated crops by fine-tuning florigen activation in crops.

\section{ARABIDOPSIS SHOOT STRUCTURE CONTROLLED BY FLORIGEN AND ANTIFLORIGEN}

Arabidopsis produce less vegetative leaves at the SAM under long-day conditions than under short-day conditions. Meristems change into floral inflorescences resulting in raceme structures with a monopodial habit, in which the main axes successively produce lateral flowers or lateral 
inflorescences with a reiterating pattern. The balance between the vegetative and inflorescence phases is well synchronized with seasonal cues such as photoperiod and vernalization (Fig. 2A) (Andrés and Coupland 2012).

In the Arabidopsis flowering time pathway, the expression of FLOWERING LOCUS C (FLC) and FRIGIDA $(F R I)$ was decreased by vernalization while and CONSTANS (CO) initiated by photoperiodic recognition promotes $F T$ expression for induction of flowering. FT is a transmissible Arabidopsis florigen that moves from the companion cells of the leaf to the SAM through the phloem sieve elements. At the shoot meristem, FT and FLOWERING LOCUS D (FD) form a complex that activates expression of flowering genes such as APETLA1 (AP1) and FUL (Abe et al. 2005). Importantly, the inflorescence meristem of the monopodium maintains the phase status by suppression of floral initiation in the central main axes, in which the antiflorigen TERMINAL FLOWER1 (TFL1) is expressed (Bradley et al. 1997). Even though TFL1 is a close paralog of $F T$, these two genes are functionally antagonistic, which means that TFL1 shows transcriptional repression of its targets through
(A)

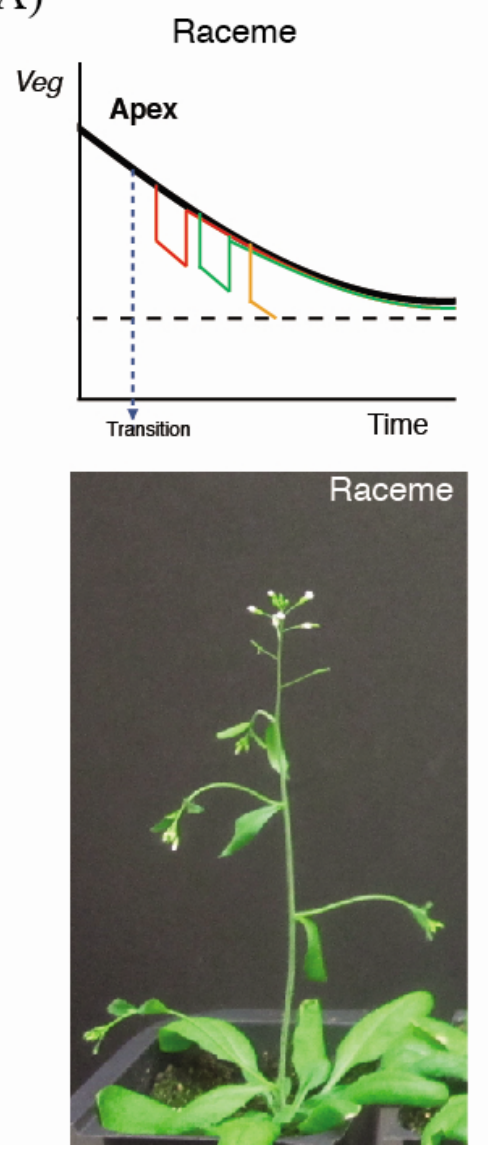

(B)
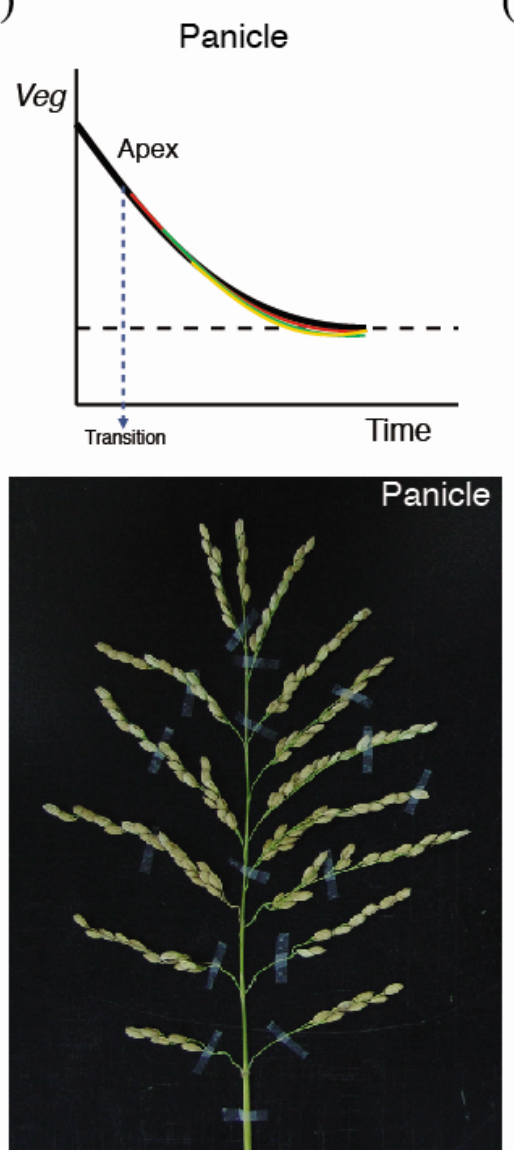

(C)
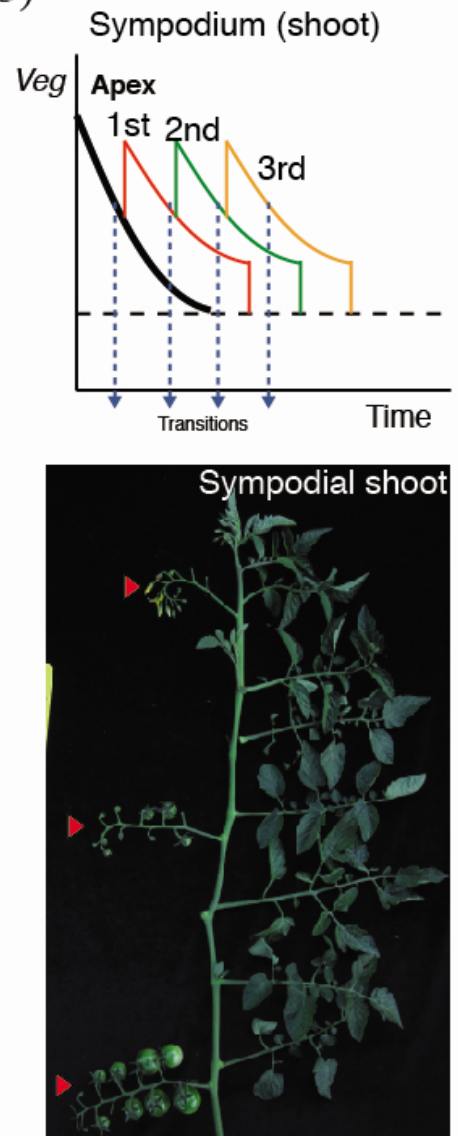

Fig. 2. Three inflorescence models with comparison of architecture and time of veg decline. (A) Raceme of Arabidopsis succesively produces lateral branches with less veg at initiation and with restored veg at elongation stage, and flowers with sufficient low veg at initiation in inflorescence. (B) Panicle of rice succesively produces lateral branches in inflorescence, which has same veg with main apex. Notably, reproductive phase is relatively shorter than that of raceme. (C) Sympodial shoot is newly initiated and gains veg, and then quickly terminates to inflorescence and flower. This pattern is reiterated in sympodial growth resulting in indeterminate growth. Red line: 1st lateral branch, Green line: 2nd lateral branch, Brown line: 3rd lateral branch, Arrow head: inflorescence. Dashed lines indicate phase transition to reproductive status.

veg: vegetativeness. 
interacting with the FD transcription factor. It is suggested that FD plays pivotal roles in the control of floral transition by interacting with FT or TFL1 (Hanano and Goto 2011). Interestingly, AP1 and LFY directly repress TFL1 transcription in lateral floral meristems and TFL1 suppresses MADS-box genes, including AP1, SOC1, $A G L 24, S V P$, and SEP4 (Liu et al. 2013). Therefore, the main axes continuously produce lateral flowers and lateral branches that reiterate the inflorescence habit (Fig. 2A).

\section{RICE SHOOT STRUCTURE CONTROLLED BY FLORIGEN PATHWAYS}

Rice is a short-day plant that enhances floral transition during the early fall season under short-day conditions. In these plants, the period of vegetative organ development is prolonged in order to produce a large number of lateral tillers during summer before floral transition (Pautler et al. 2013). After the transition stage, the SAM is transformed into an inflorescence meristem, which increases size via cell proliferation, and then forms a panicle structure that bears branch meristems. The branch meristems subsequently undergo consecutive development of spikelet meristems, which terminate in a flower (Fig. 2B) (Tanaka et al. 2013).

The OsGI (Oryza sativa GIGANTEA) in the rice flowering pathway is highly conserved with Arabidopsis $G I$, which responds to photoperiod. As a downstream gene of OsGI, Hdl, which encodes a homolog of Arabidopsis $\mathrm{CO}$, promotes flowering under short days but represses flowering by regulating $H d 3 a$ expression under long days (Hayama et al. 2003). Although rice accelerates floral induction under short days, the genes involved in each step of the mechanism are highly conserved between rice and Arabidopsis; they have, however, functionally diverged during their respective speciations (Hayama et al. 2003). Additionally, Ehd1, a B-type response regulator, induces expression of the rice florigens $H d 3 a$ and RFTl under short-day conditions, which is functionally independent of the pathway containing Hdl (Doi et al. 2004). GHD7 (which is involved in determining grain number, plant height, and heading date), which encodes a CCT-domain protein represses the transcription of Ehd 1 under long-day conditions. In rice, the Ghd7-Ehd1-Hd3a pathway is functionally distinct from that in Arabidopsis, as Ehdl has no ortholog in the Arabidopsis genome (Xue et al. 2008; Lu et al. 2012).

The rice florigen $\mathrm{Hd} 3 \mathrm{a}$ is expressed in the leaf vasculature and the Hd3a protein moves systemically to the SAM as a mobile hormone that regulates the phases of plant growth according to photoperiod. In the shoot apical cells, Hd3a interacts with 14-3-3 proteins, forming a complex that is translocated to the nucleus and binds with the Oryza sativa (Os)FD1 transcription factor, a rice homolog of FD. The hexamerous complex is formed by a doubling of Hd3a:14-3-3:OsFD1, referred to as the "florigen activation complex (FAC)," that induces transcription of OSMADS15, an ortholog of $A P 1$, leading to determination of the floral identity of meristems (Taoka et al. 2011). A further rice antiflorigen RICE CENTRORADIALIS4 (RCN4) enhances panicle branches when it is ectopically expressed but $R C N$ knock down lines produced considerably smaller panicles in rice because of the reduced number of branches. Rice orthologs of SEP4, AGL24, SOC1, and SVP regulate panicle structure because knock down lines of these genes increase the number of panicle branches and also show ectopic expression of RCN4 (Liu et al. 2013). These results indicate that panicle branching is determined by rice orthologs of SEP4, AGL24, SOC1, and SVP through suppressing antiflorigen $R C N s$ in the lateral meristems of rice.

\section{SYMPODIAL GROWTH AND TOMATO FLORIGEN}

Flowering of tomato is dependent not on day length but on the total daily light integrals (light dose) during shoot growth. Seven to twelve leaves were produced by the primary shoot meristem (PSM) and then undergoes a transition to reproductive growth, producing a lateral sympodial inflorescence meristem that subsequently give rise to a single lateral inflorescence. This process is repeated more than six times in the tomato inflorescence (Lifschitz and Eshed 2006). 
In the axil of the last leaf on the PSM, a specialized axillary meristem, referred to as the sympodial shoot meristem (SYM), is initiated, and this grows upright as the new main shoot. The SYM consistently produces three leaves following transition from the vegetative meristem to the floral meristem, and then a new SYM subsequently forms in the axil of the last leaf produced by the previous SYM. This process reiterates such SYMs develop three leaves and a terminal inflorescence. Tomato SYMs indeterminately develop compound vegetative shoots, which is referred to as the perennial sympodial habit (Fig. 2C) (Park et al. 2012).

Approximately 90 years ago, the naturally occurring $s p$ mutant was shown to modify "indeterminate" plants into a new "determinate" growth form that has been bred and exploited in large-scale tomato farming associated with the tomato processing industry (Yeager 1927). However, it takes six decades to clone SELF PRUNING (SP) gene after the discovery of the $s p$ mutant. $S P$ gene is a homolog of the A. thaliana TFL1 gene, which encodes a repressor of flowering, and is an antiflorigen in the CETS protein family (Pnueli et al. 1998). Interestingly, $S P$ is not expressed in the center of the PSM, which differs from species with a monopodial habit; however, $S P$ transcripts are located in the initial region during outgrowth of the SYM and axillary meristems after floral transition at the PSM. SP is only expressed in non-growing axillary meristems, including the SYM. Taken together, the observed expressions of $S P$ indicate that this gene functions in the out-growth of both sympodial and all other axillary meristems (Thouet $e t$ al. 2008).

SFT (SINGLE FLOWER TRUSS), the tomato ortholog of $F T$, encoding tomato florigen induces flowering in dayneutral tomato. This florigen acts as systemic signal that enhances the growth and transition of vegetative AM. The tomato $\mathrm{FAC}$ is functionally and physically conserved with that of rice FAC, which comprises SP-interacting G-BOX, a homolog of $\mathrm{FD}$, encoding a basic region/leucine zipper (bZIP) transcription factor that interacts with 14-3-3 bound physically to SFT at the SAM (Pnueli et al. 2001; Park et al. 2014b). Additionally, uniflora (uf), jointless (j), macrocalyx $(m c)$, and compound inflorescence $(s)$ delay flowering time in tomato, indicating that $U F, J, M C$, and $S$ play a functional role in regulating flowering time.

\section{OVERDOMINACE OF $s f t /+$ AND FLORIGEN DOSAGE EFFECT}

Zamir and his colleague reported that overdominant (ODO) quantitative trait loci (QTL) are highly correlated with reproduction traits in tomato, suggesting that ODOQTL have been naturally selected to confer fitness through heterozygous advances in evolution, resulting in a new balance between the diversity and opposing isolation needs among gene pools. ODO-QTLs may be domesticated by humans to improve agricultural crop yield, which is termed as heterosis (Semel et al. 2006; Lippman et al. 2007).

As a first example of a single ODO gene for yield, the heterozygous mutant of tomato florigen $(s f t /+)$ drives heterosis for yield. The overdominance is derived from the suppression of sympodial shoot growth termination mediated by the $s p$ mutant, an antagonist of $S F T$. This indicates that $s f t$ heterozygosity $(s f t+)$ causes weak semidominant delays in flowering in both primary and side shoots in an $s p$ mutant background. This in turn suggests that single heterozygous mutations may improve productivity in other agricultural organisms by modification of flowering time and plant structure (Krieger et al. 2010; Jiang et al. 2013). Further, molecular quantification by transcriptome profiling of shoot meristems revealed that the transitional meristem (TM) of $s f t /+s p$ displays a molecular status between that of the TM of the $s f t s p$ and $s p$ mutants, and that the SYM of $s f t /+s p$ displays a molecular status between that of the TM of the wild type and $s p$ mutant. This indicates that tomato yield overdominance results from the dosage sensitivity of the florigen pathway that can control shoot structure (Veitia 2007; Jiang et al. 2013).

Park et al. (2014), subsequently identified a way to optimize tomato yield in the field by modifying the balance between florigen activation and its suppression. This result was molecularly supported by a mathematical model suggesting that dominant negative interactions might give rise to heterotic responses and a biochemical model of gene dosage effects might be evaluated in macromolecular complexes by dominant negative interactions (Veitia and 
Vaiman 2011). On the basis of the gene dosage effects and dominant negative interactions, the tomato FAC was used for manipulating a gradient of flowering signal levels by creating a series of single- and double-mutant heterozygous genotypes in the $s p$ mutant background (Veitia 2007; Park et al. 2014b). Because shoot determinacy and sympodial cycling are progressively changed according to the range of florigen activation dosage effects, the growths of heterozygote mutants shows a continuum of shoot architectures from determinate to indeterminate sympodial shoot growth, which resulted in translations to a continuum of yields. ssp-2129/+ sft-1906/+ among the heterozygote mutants produced the highest yield with optimal plant architecture under ideal field condition. These results suggest a general model for increasing productivity, in which optimum yields are achieved by balancing flowering signals that shift determinate growth to indeterminate growth (Fig. 3). The model suggests that a similar gradient of flowering signals and yields could be achieved through other means, indicating that alleles of the florigen pathway genes comprise a flexible toolkit to develop high-yielding hybrids (Park et al. 2014b).

(A)

$s p$

Determinate (D)

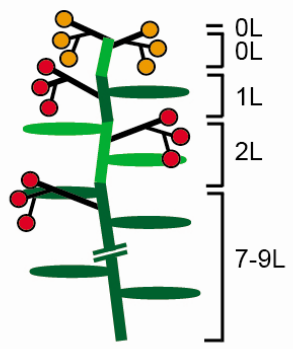

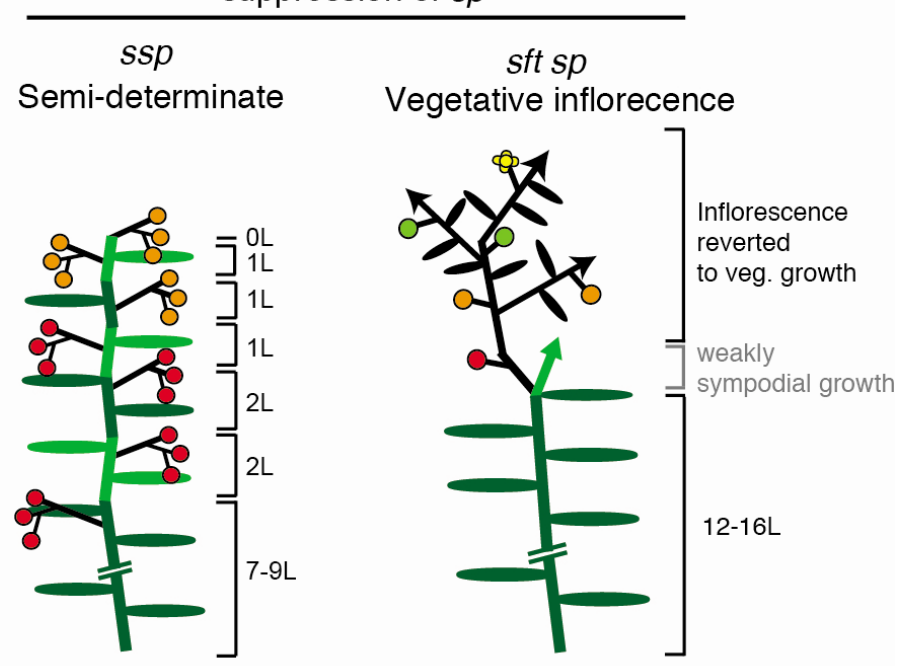

suppression of $s p$

(B)

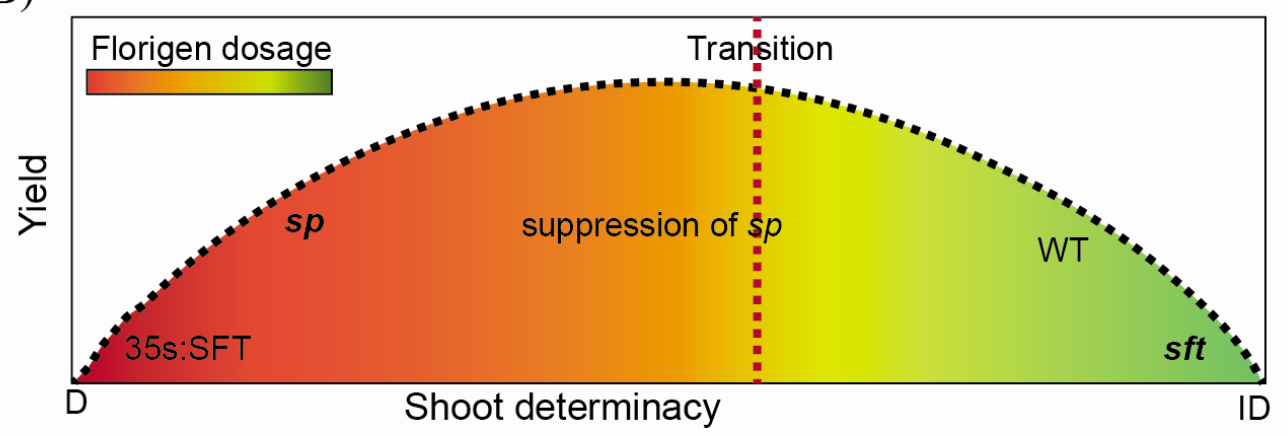

Fig. 3. Diagrams depicting overdominance with florigen mutants (A) and dosage model of florigen activation and yield fitness in tomato (B). (A) Diagrams representing additive suppression of determination, indicating yield overdominance by plant architecture (suppression of $s p$ ). The black bars represent the inflorescence. Alternating dark green and light green bars reflect successive sympodial shoots. Colored circles represent maturing fruits. (B) Model of tomato yield in relation to the range of floral activation and shoot determinacy. Both extremely strong and weak florigen activation conditions such as $35 S$ :SFT and $s f t$ mutant yield very low but moderate genotypes potentially produce the highest yield. Red dashed lines indicate transition from determinate to indeterminate shoot growth.

veg: vegetativeness, SFT: SINGLE FLOWER TRUSS, WT: wild type. 
This model is applicable to other crops. For example, DETERMINATE STEM1 (Dt1), a homolog of TFL1, is expressed in soybean SAM and $d t 1 / d t 1$ strongly suppresses shoot growth resulting in the determinate habit. In contrast, the growth of $D t 1 / D t 1$ is similar to that of the wild type with an indeterminate growth habit. The hybrid $d t 1$ mutant (Dt1/dt1) shows a distinct semi-determinate growth phenotype, indicating that single $D t 1$ has a partially dominant function in shoot growth at the apices (Liu et al. 2010; Tian et al. 2010). Additionally, domain-expressing Dt2, a soybean homolog of $A P 1$, suppresses $D t 1$, which is functionally conserved with Arabidopsis AP1. Nine independent lines overexpressing $D t 2$ grow with an unusual gradual apical stem termination phenotype, which is associated with the degree of expression (Liu et al. 2010). These lines are a potential genetic source that could be used to fine-tune and optimize yields of soybean in certain areas (Ping et al. 2014). Therefore, the universal florigen flowering pathway provides a new opportunity for breeders to generate sensitive and gradual shoot growth variations in crops postdomestication by using advanced bioengineering technology.

\section{MANIPULATION OF DOSAGE EFFECT AND GENOME EDITING}

The phenotypic basis of ODO may be an intermediate or moderate phenotype compared with that of both parents, but provides QTLs for high yield. The dosage model of tomato florigen activation represents remarkable gradual variations in tomato shoot determinacy. This can be described in term of the dominant negative interactions of FAC giving rise to a dosage effect of the complex under heterotic responses. On the basis of this model, the optimal balance of flowering signals leads to the highest yield at the shift from determinate growth to indeterminate growth (Fig. 3) (Veitia 2007; Park et al. 2014b).

In sunflower, a frameshift mutation in Helianthus annuus FT1 (HaFT1), a homolog of FT, induces a dominant-negative effect on developmental timing and caused a flowering QTL by interfering partially with flower enhancement by HaFT4, which are affected by the transcripts (Blackman et al. 2010). In oil palm, SHELL, a homolog of the MADS-box gene SEEDSTICK, is another overdominance gene. Sh/Sh (dura) fruit have a lignified thick shell surrounding the kernel but $s h / s h$ (pisifera) gives rise to a shell-less fruit form. The hybrid palms $(S h / s h)$ have a thin shelled fruit form (tenera) that result in a considerably higher yield because intermediate fruit forms give rise to a distinct fiber ring and oil palm seed with a thin shell (Singh et al. 2013).

With regard to the development of reproductive architecture in crops, weak mutant alleles present the potential for enhancement of yield QTL, because strong gain or loss of function mutations often result in severe yield traits with negative effects in plant architecture. For example, the null fea 2 mutant allele confers strong development of fasciated ears with increased kernel row number (KRN; about approximately 30); however, the ear is short with a flattened tip resulting less yield potential. Nevertheless, a weak fea 2 allele can increase KRN with high yield potential, suggesting that the CLAVATA receptor-like protein FASCIATED EAR2 (FEA2) locus is related to the QTL for maize KRN (Bommert et al. 2013). Additionally, FASCIATED EAR3 (FEA3), a leucine-rich-repeat receptor, controls stem cell proliferation. The null fea 3 mutant produces fasciated ears and increased KRN. In contrast, weak alleles of $f e a 3$ enhance yield traits (Je et al. 2016).

In tomato, a forked type inflorescence provides structural dominance for fruit yield improvement with a balance between vegetative and reproductive tissue. compound inflorescence ( $s$ ), a homologue mutant of WUSCHEL HOMEOBOX 9 (WOX9), develops highly branched inflorescences bearing hundreds of flowers but normal or less fruit setting because of the excess flower production in each inflorescence (Lippman et al. 2008). The hybrid tomato $S / s$ produces a high proportion of forked inflorescences, indicating that weak or intermediate branched inflorescences in $S / s$ may lead to high yield production (Jiang et al. 2013).

Plant structural variations can be used for crop improvement in breeding programs. Artificial variations regulating plant architecture, such as florigen mutants, can be isolated in breeding populations by whole-genome sequencing or by Targeting-Induced Local Lesions in Genomes (Minoia 
et al. 2010; Pérez-de-Castro et al. 2012). The CRISPR/Cas9 gene editing system, which is a targeted mutagenesis technology, will lead to future developments in plant breeding because this technology is very simple and broadly applicable to many crops. It will also provide a potential alternative to genetically modified organisms.

The CRISPR/Cas9 genome editing system was originally derived from the bacterial immune system. sgRNAs (single guide RNAs) are targeted to specific locations in the plant genome and Cas9 nuclease recognizes the sgRNA and protospace adjacent motif (PAM) sequences, resulting in a double-stranded break (Sander and Joung 2014; Khatodia et al. 2016). Using these engineered nucleases, the CRISPR/ Cas9 system has been broadly expanded to gene knockout, DNA replacement, multiplex editing, and transcription modulation in plants (Sander and Joung 2014). Therefore, the CRISPR- Cas9 system is available for breeders to generate mutants in plant structural QTLs and in multiple loci, inducing dominant negative effects in hybrids and by generating deletions in promoter regions to produce variable-strength alleles. First, the promoter of genes associated with the florigen pathway can be targeted by multiple sgRNAs to generate many combinations of deletions in the promoter. Some deletions, including suppressor cis-regulatory elements, are expected to give rise to gene enhancement. Other deletions in enhancer cis-regulatory elements will suppress expression. The introduction of deletions in promoters will provide breeders with numerous varieties regarding the sequential gradient of gene expression. Second, components in multiple florigen pathways can be mutated using the CRISPR/Cas9 system. Genetic interactions among the mutants in the components may reveal flowering time variations. Additionally, multiple heterozygous mutants in protein complexes will show partial dominate phenotypes resulting in gradual shoot determinacy, which will be valuable for fine-tuning and optimizing crop yield. Third, the interactome of florigen, plant structural QTLs, and ODO QTLs will be very useful sources for systemic breeding approaches by exploring dosage effect or sensitivity due to variations in gene complexes. The Arabidopsis interactome (http://bar.utoronto.ca/interactions/ cgi-bin/arabidopsis_interactions_ viewer.cgi) and the predicted rice interactome (http:// bis.zju.edu.cn/prin/) are available to validate the protein complex of florigen, plant structural QTLs, and ODO QTLs by transient bimolecular fluorescence complementation assay and the targeting CRISPR/Cas9 system. Positive negative effects and dosage effects derived from complex variations can thus be taken advantage of for enhancing the reproductive fitness of crops.

\section{CONCLUSION}

The phosphatidylethanolamine-binding protein gene family including florigens and antiflorigens have evolved antagonistic functions in the regulation of flowering (Schoentgen et al. 1987; Kobayashi et al. 1999). Leafinduced florigen signals promote systemic acceleration of flowering on apical and lateral meristems. Antiflorigens expressed in local domains at temporal status directly repress flowering in lateral meristems (Shalit et al. 2009). Interestingly, some florigen homologs expressed in the leaf have been systemically functionalized for repressing flowering and responding to vernalization in certain species such as sugar beet and sunflower (Blackman et al. 2010; Pin et al. 2010).

In tomato, the balance model of mobile florigen (SFT) and local antiflorigen (SP) suggests that the local ratio of $\mathrm{SFT} / \mathrm{SP}$ determines flowering transition and termination at primary, sympodial, and lateral meristems. The ratio is adjusted to the reiterative growth and termination cycles, referred to as the sympodial index, in SYM (Lifschitz et al. 2006; Shalit et al. 2009). In Arabidopsis, florigen and antiflorigen are functionally antagonistic and may compete in the role of transcription regulation of floral genes (Hanano and Goto 2011). In rice, the florigen activation model suggests that mobile florigens form a complex by interacting with OsFD1 and 14-3-3 in the nucleus of SAM cells and activate OsMADS15 and floral identity genes (Taoka et al. 2011). This FAC model is physically and functionally conserved in the tomato system, indicating that basic florigen function might be mediated via the FAC in flowering plants.

The dosage model of FAC suggests that the dosage of flowering signals can be manipulated by generating genetic 
combinations of the FAC component modifiers. This is because dosage effects might result from dominant negative interactions or expressional and functional variation. In tomato, this model has been successfully applied for yield improvement in a single plant and strongly suggests that this approach represents a flexible toolkit for developing high-yielding hybrids. This model might be universally applicable to other species such as rice and soybean (Park et al. 2014b). For example, a recent GWAS study on rice heterosis has indicated that $\mathrm{Hd} 3 \mathrm{a}$ is one of the effector genes for yield enhancement in rice hybrids (Huang et al. 2016).

Finally, genome editing provides a very useful toolkit for plant breeding, since with the CRISPR/Cas9 technique, breeders can potentially generate considerable variation in the gradient expression or function of QTL genes, particularly targeted single and multiple gene mutations, and deletion of $c i s$-regulatory elements. Consequently, the integration of studies focusing on the dosage effect of florigen activation and ODO QTLs, and judicious use of the CRISPR/Cas9 system will constitute a promising crop breeding strategy for future crop improvement.

\section{ACKNOWLEDGEMENTS}

We thank Z. Lippman (Cold Spring Harbor Laboratory) for discussions. This work was supported by the NextGeneration BioGreen 21 Program (PMBC, PJ01191201) to S.J.P., and (PMBC, PJ01191202) to J.H.B.

\section{REFERENCES}

Abe M, Kobayashi Y, Yamamoto S, Daimon Y, Yamaguchi A, Ikeda Y, et al. 2005. FD, a bZIP protein mediating signals from the floral pathway integrator FT at the shoot apex. Science 309: 1052-1056.

Andrés F, Coupland G. 2012. The genetic basis of flowering responses to seasonal cues. Nat. Rev. Genet. 13: 627-639.

Blackman BK, Strasburg JL, Raduski AR, Michaels SD, Rieseberg LH. 2010. The role of recently derived FT paralogs in sunflower domestication. Curr. Biol. 20:
629-635.

Bommert P, Nagasawa NS, Jackson D. 2013. Quantitative variation in maize kernel row number is controlled by the FASCIATED EAR2 locus. Nat. Genet. 45: 334-337.

Bradley D, Ratcliffe O, Vincent C, Carpenter R, Coen E. 1997. Inflorescence commitment and architecture in Arabidopsis. Science 275: 80-83.

Doi K, Izawa T, Fuse T, Yamanouchi U, Kubo T, Shimatani $\mathrm{Z}$, et al. 2004. Ehd1, a B-type response regulator in rice, confers short-day promotion of flowering and controls FT-like gene expression independently of Hd1. Genes. Dev. 18: 926-936.

Grillo MA, Li C, Hammond M, Wang L, Schemske DW. 2013. Genetic architecture of flowering time differentiation between locally adapted populations of Arabidopsis thaliana. New Phytol. 197: 1321-1331.

Hanano S, Goto K. 2011. Arabidopsis TERMINAL FLOWER1 is involved in the regulation of flowering time and inflorescence development through transcriptional repression. Plant Cell 23: 3172-3184.

Hayama R, Yokoi S, Tamaki S, Yano M, Shimamoto K. 2003. Adaptation of photoperiodic control pathways produces short-day flowering in rice. Nature 422: 719-722.

Huang X, Yang S, Gong J, Zhao Q, Feng Q, Zhan Q, et al. 2016. Genomic architecture of heterosis for yield traits in rice. Nature 537: 629-633.

Izawa T. 2007. Adaptation of flowering-time by natural and artificial selection in Arabidopsis and rice. J. Exp. Bot. 58: 3091-3097.

Je BI, Gruel J, Lee YK, Bommert P, Arevalo ED, Eveland AL, et al. 2016. Signaling from maize organ primordia via FASCIATED EAR3 regulates stem cell proliferation and yield traits. Nat. Genet. 48: 785-791.

Jiang K, Liberatore KL, Park SJ, Alvarez JP, Lippman ZB. 2013. Tomato yield heterosis is triggered by a dosage sensitivity of the florigen pathway that fine-tunes shoot architecture. PLoS Genet. 9: e1004043.

Khatodia S, Bhatotia K, Passricha N, Khurana SM, Tuteja N. 2016. The CRISPR/Cas genome-editing tool: application in improvement of crops. Front. Plant Sci. 7: 506.

Kobayashi Y, Kaya H, Goto K, Iwabuchi M, Araki T. 1999. A pair of related genes with antagonistic roles in mediating flowering signals. Science 286: 1960-1962.

Krieger U, Lippman ZB, Zamir D. 2010. The flowering gene SINGLE FLOWER TRUSS drives heterosis for yield in 
tomato. Nat. Genet. 42: 459-463.

Lifschitz E, Eshed Y. 2006. Universal florigenic signals triggered by FT homologues regulate growth and flowering cycles in perennial day-neutral tomato. J. Exp. Bot. 57: 3405-3414.

Lifschitz E, Eviatar T, Rozman A, Shalit A, Goldshmidt A, Amsellem Z, et al. 2006. The tomato FT ortholog triggers systemic signals that regulate growth and flowering and substitute for diverse environmental stimuli. Proc. Natl. Acad. Sci. U.S.A. 103: 6398-6403.

Lippman ZB, Cohen O, Alvarez JP, Abu-Abied M, Pekker I, Paran I, et al. 2008. The making of a compound inflorescence in tomato and related nightshades. PLoS Biol. 6: e288.

Lippman ZB, Semel Y, Zamir D. 2007. An integrated view of quantitative trait variation using tomato interspecific introgression lines. Curr. Opin. Genet. Dev. 17: 545-552.

Liu B, Watanabe S, Uchiyama T, Kong F, Kanazawa A, Xia $\mathrm{Z}$, et al. 2010. The soybean stem growth habit gene Dt1 is an ortholog of Arabidopsis TERMINAL FLOWER1. Plant Physiol. 153: 198-210.

Liu C, Teo ZW, Bi Y, Song S, Xi W, Yang X, et al. 2013. A conserved genetic pathway determines inflorescence architecture in Arabidopsis and rice. Dev. Cell 24: 612-622.

Lu L, Yan W, Xue W, Shao D, Xing Y. 2012. Evolution and association analysis of Ghd7 in rice. PLoS One 7: e34021.

Minoia S, Petrozza A, D'Onofrio O, Piron F, Mosca G, Sozio $\mathrm{G}$, et al. 2010. A new mutant genetic resource for tomato crop improvement by TILLING technology. BMC Res. Notes. 3: 69.

Nakamichi N. 2015. Adaptation to the local environment by modifications of the photoperiod response in crops. Plant Cell Physiol. 56: 594-604.

Park SJ, Eshed Y, Lippman ZB. 2014a. Meristem maturation and inflorescence architecture--lessons from the Solanaceae. Curr. Opin. Plant Biol. 17: 70-77.

Park SJ, Jiang K, Schatz MC, Lippman ZB. 2012. Rate of meristem maturation determines inflorescence architecture in tomato. Proc. Natl. Acad. Sci. U.S.A. 109: 639-644.

Park SJ, Jiang K, Tal L, Yichie Y, Gar O, Zamir D, et al. 2014b. Optimization of crop productivity in tomato using induced mutations in the florigen pathway. Nat. Genet. 46: 1337-1342.
Pautler M, Tanaka W, Hirano HY, Jackson D. 2013. Grass meristems I: shoot apical meristem maintenance, axillary meristem determinacy and the floral transition. Plant Cell Physiol. 54: 302-312.

Pérez-de-Castro AM, Vilanova S, Cañizares J, Pascual L, Blanca JM, Díez MJ, et al. 2012. Application of genomic tools in plant breeding. Curr. Genomics 13: 179-195.

Pin PA, Benlloch R, Bonnet D, Wremerth-Weich E, Kraft T, Gielen JJ, et al. 2010. An antagonistic pair of FT homologs mediates the control of flowering time in sugar beet. Science 330: 1397-1400.

Ping J, Liu Y, Sun L, Zhao M, Li Y, She M, et al. 2014. Dt2 is a gain-of-function MADS-domain factor gene that specifies semideterminacy in soybean. Plant Cell 26: 2831-2842.

Pnueli L, Carmel-Goren L, Hareven D, Gutfinger T, Alvarez J, Ganal M, et al. 1998. The SELF-PRUNING gene of tomato regulates vegetative to reproductive switching of sympodial meristems and is the ortholog of CEN and TFL1. Development 125: 1979-1989.

Pnueli L, Gutfinger T, Hareven D, Ben-Naim O, Ron N, Adir $\mathrm{N}$, et al. 2001. Tomato SP-interacting proteins define a conserved signaling system that regulates shoot architecture and flowering. Plant Cell 13: 2687-2702.

Sander JD, Joung JK. 2014. CRISPR-Cas systems for editing, regulating and targeting genomes. Nat. Biotechnol. 32: 347-355.

Schoentgen F, Saccoccio F, Jollès J, Bernier I, Jollès P. 1987. Complete amino acid sequence of a basic $21-\mathrm{kDa}$ protein from bovine brain cytosol. Eur. J. Biochem. 166: 333-338.

Semel Y, Nissenbaum J, Menda N, Zinder M, Krieger U, Issman $\mathrm{N}$, et al. 2006. Overdominant quantitative trait loci for yield and fitness in tomato. Proc. Natl. Acad. Sci. U.S.A. 103: 12981-12986.

Shalit A, Rozman A, Goldshmidt A, Alvarez JP, Bowman JL, Eshed Y, et al. 2009. The flowering hormone florigen functions as a general systemic regulator of growth and termination. Proc. Natl. Acad. Sci. U.S.A. 106: 83928397.

Singh R, Low ET, Ooi LC, Ong-Abdullah M, Ting NC, Nagappan J, et al. 2013. The oil palm SHELL gene controls oil yield and encodes a homologue of SEEDSTICK. Nature 500: 340-344.

Song YH, Ito S, Imaizumi T. 2013. Flowering time regulation: photoperiod- and temperature-sensing in leaves. Trends 
Plant Sci. 18: 575-583.

Tanaka W, Pautler M, Jackson D, Hirano HY. 2013. Grass meristems II: inflorescence architecture, flower development and meristem fate. Plant Cell Physiol. 54: 313-324.

Taoka K, Ohki I, Tsuji H, Furuita K, Hayashi K, Yanase T, et al. 2011. 14-3-3 proteins act as intracellular receptors for rice Hd3a florigen. Nature 476: 332-335.

Thouet J, Quinet M, Ormenese S, Kinet JM, Périlleux C. 2008. Revisiting the involvement of SELF-PRUNING in the sympodial growth of tomato. Plant Physiol. 148: 61-64.

Tian Z, Wang X, Lee R, Li Y, Specht JE, Nelson RL, et al. 2010. Artificial selection for determinate growth habit in soybean. Proc. Natl. Acad. Sci. U.S.A. 107: 8563-8568.

Veitia RA. 2007. Exploring the molecular etiology of dominant-negative mutations. Plant Cell 19: 3843-3851.

Veitia RA, Vaiman D. 2011. Exploring the mechanistic bases of heterosis from the perspective of macromolecular complexes. FASEB J. 25: 476-482.

Xue W, Xing Y, Weng X, Zhao Y, Tang W, Wang L, et al. 2008. Natural variation in Ghd7 is an important regulator of heading date and yield potential in rice. Nat. Genet. 40: 761-767.

Yeager AF. 1927. Determinate growth in the tomato. J. Hered. 18: 263-265. 